\title{
Full-Vectorial Description of the Light Guidance in Anisotropic Photonic Liquid Crystal Fibers
}

\author{
K.A. RutKowska ${ }^{a, *}$ And L.W. WeI ${ }^{b}$ \\ ${ }^{a}$ Faculty of Physics, Warsaw University of Technology, Koszykowa 75, 00-662 Warsaw, Poland \\ ${ }^{b}$ Institute of Micromechanics and Photonics, Warsaw University of Technology \\ A. Boboli 8, 02-525 Warsaw, Poland \\ In this paper we present our theoretical approach for the description of the light guidance in photonic \\ liquid crystal fibers. In particular, we focus on the numerical methods allowing for a full implementation of the \\ liquid crystals properties (i.e., including their optical anisotropy and molecular orientation), with a final target \\ in characterizing photonic liquid crystal fibers with accessible computational effort. For this purpose suitable \\ analytical formulae required for a full-vectorial description of the optical modes in photonic liquid crystal fibers \\ have been derived. In addition, computational schemes allowing for numerical implementation of theoretical \\ formulations (with the use of the finite-difference scheme) have been developed, validated and optimized. Their \\ numerical convergence has been checked for different structures, as well as for different input parameters (e.g., \\ grid-size). Obtained results have been compared to those analytically calculated, known from literature and/or \\ got with use of commercial software. Moreover, the implemented schemes have been examined in accordance with \\ experimental tests performed on the photonic liquid crystal fiber of interest.
}

PACS: $02.60 . \mathrm{Cb}, 02.70 . \mathrm{Bf}, 42.25 . \mathrm{Bs}, 42.70 . \mathrm{Qs}, 42.81 . \mathrm{Wg}, 42.70 . \mathrm{Df}$

\section{Introduction}

Photonic crystal fibers (PCFs) are the special class of 2D photonic crystal structures with cylindrical symmetry, extended homogeneously over the third dimension (i.e., propagation axis) [1-3]. As shown in Fig. 7a, typical cross-section of PCF can be divided into two regions - the circular core and the annular cladding. The latter generally consists of periodically distributed air-holes (forming a photonic lattice), while the core region can either be formed from solid materials as in conventional fibers, or remain hollow in some designs (not presented here). Possible modification of the fiber geometry (e.g., change in size and location of the air-holes) gives additional degree of freedom in engineering the characteristics of the optical fibers. It determines that in addition to the index-guiding mechanism (defined also as a modified total internal reflection, mTIR) - when light is spatially localized in regions with higher refractive indices (e.g., in a solid core of the holey microstructured optical fibers) PCFs can also propagate light as an effect of the photonic bandgap (PBG) guiding (e.g., in a hollow-core PCFs). Infiltration of the air-holes with gaseous and liquid media leads to even more extraordinary properties of PCFs. Specifically, the concept of filling them with liquid crystals (LCs), resulting thus in a new type of optical fiber, has recently gained significant amount of the scientific attention [4-8].

These particular photonic structures are often referred to as photonic liquid crystal fibers (PLCFs) [4, 5], liquid crystal-photonic crystal fibers (LC-PCFs) [6], or liquid crystal-photonic bandgap fibers [7]. LCs are anisotropic, and, in most cases, their averaged refractive indices

* corresponding author; e-mail: kasia@if.pw.edu.pl are higher than that of silica. The infiltration with LCs allows to convert an initially index-guiding PCF with a solid (glass) core to a PBG-guiding PLCF. However, by substituting the silica substrate with materials with higher refractive indices (e.g., lead-bismuth-silicate glass [8]) or choosing $\mathrm{LC}$ with lower refractive index (e.g., $1550 \mathrm{LC}$ mixture [9]), it is also possible to achieve index-guiding mechanism in PLCF of the same geometry.

Furthermore, the refractive indices of LCs can also be engineered and/or dynamically adjusted so that both guiding mechanisms are possible within the same PLCF structure [5]. High tunability of optical properties of PLCFs, achieved by applying external factors like temperature, strain, pressure, electric or/and magnetic fields, as well as by the light-beam itself (e.g., when nonlinear effects are considered), brings various potential applications to the field of fiber optics $[4-8,10,11]$. However, it has to be underlined that owing to their quasi-crystalline and non-uniform nature, LCs introduce further challenge in theoretical and experimental characterization of the corresponding fiber optics elements and components.

\section{Model development}

Conventionally, the most well-known and the simplest model for the optical fiber characterization is the analytical scalar-field formulation with linearly polarized (LP) field approximation in which the vector fields are decoupled into individual scalar fields [12]. It provides fairly straightforward and accurate characterization of practical optical and telecommunication fibers (i.e., step-index geometries with small index-contrast), but fails for more complicated structures (for which a vector-field formulation is required). In particular, when microstructured optical fibers (MOFs) and photonic crystal structures are incorporated, analytical solutions are difficult to obtain, if not impossible. 
Fortunately, nowadays, thanks to the advance of computer science and computational physics, various types of numerical methods for characterization of PCFs could be developed [13]. Among possible modeling schemes, the finite difference method in frequency domain (FDFD) has been chosen in this work. In particular, both scalar-field and vector-field FDFD schemes have been implemented to characterize the PLCFs of interest. Specifically, taking into consideration the computational accessibility and efficiency, a special form of a vector-field FDFD has been formulated. Proposed solution is based on the scheme shown in [14], while extending its use to optical fibers with more general material anisotropy.

\subsection{Finite difference method in frequency domain}

Typically, FDFD schemes involve all four Maxwell equations which relate vector fields $(E, H, D, B)$ with their derivatives $(\partial x, \partial y, \partial z, \partial t)$. While only fields of a fixed angular frequency $\left(\omega_{0}\right)$ are considered (as it is in the frequency domain methods), the time derivative results in direct multiplication by $-\mathrm{i} \omega_{0}$. Moreover, based on the mode theory for electromagnetic fields in optical waveguides (where modes are mathematically defined as the possible solutions of the wave equations with constant field distributions along the propagation axis), the $z$-derivative is also reduced to direct multiplication (by $\mathrm{i} \beta$, where $\beta$ is propagation constant). Eventually, the remaining $x$ - and $y$-derivatives are approximated by finite-difference formulae and with all derivatives deduced, the four Maxwell equations (represented in the form of wave equations, where second-order derivatives with respect to the transverse spatial coordinates appear) may be solved numerically for a specific geometry and optical properties of the medium [15-18].

On the other hand, in [14] authors first proposed a more compact FDFD scheme incorporating Yee's staggered mesh incorporated (which is widely used in FDTD schemes owing to its effectiveness) [19] to study the modal characteristics of MOFs. Numerous publications followed such FDFD scheme and extended its use to even more general fiber geometries [20-22].

\subsection{Scalar-field FDFD}

When describing modes propagating in optical waveguides, the Maxwell equations are very often introduced in the further reduced form where the vector nature of the electric and magnetic field/induction is dropped, resulting thus in the scalar wave formulation. The latter is sufficient when there is no coupling among electric and magnetic field components, allowing thus for the tensor notation of the $E$ - and $H$-fields to be neglected. For isotropic media, within a linear regime, and with a certain time and propagation direction dependence of the fields (as mentioned in previous section), the scalar-field eigenvalue problem is described by the following equation:

$$
\left(\frac{\partial^{2}}{\partial x^{2}}+\frac{\partial^{2}}{\partial y^{2}}+k_{0}^{2} \varepsilon\right) E_{\mathrm{T}}=\beta^{2} E_{\mathrm{T}},
$$

where $\varepsilon$ is (relative) electric permittivity and the $T$ subscript denotes the transverse components of the electric field. For the magnetic field an eigenvalue problem equation takes identical form (with $H_{\mathrm{T}} \equiv E_{\mathrm{T}}$ ).
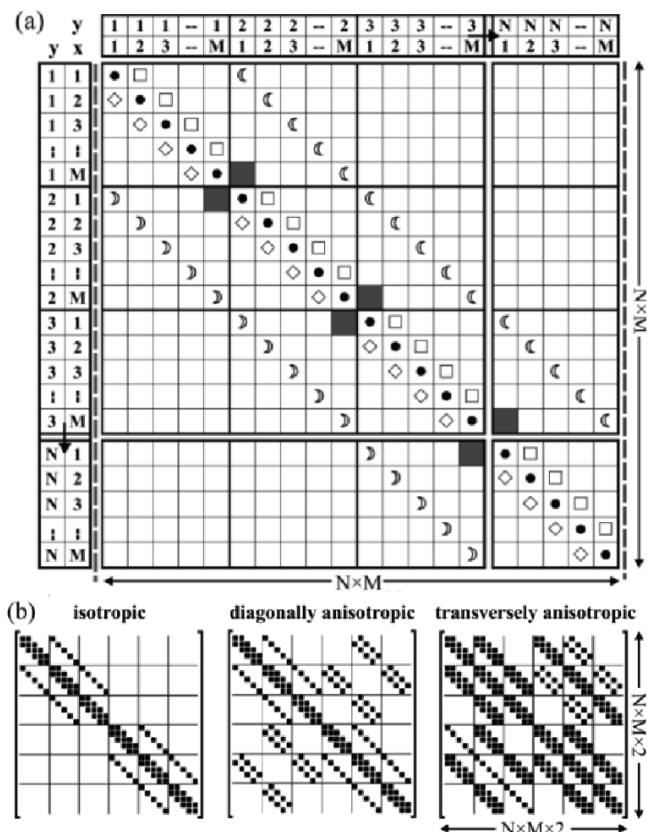

Fig. 1. Use of the three-point FD for discretization of the scalar-field eigenvalue problem with corresponding global matrix [whose elements are given by Eq. (2)] (a). In the matrix arrangement applied, the horizontal borders (dashed lines) represent the boundaries in the $y$-direction, while the boundaries in the $x$-direction are colored in grey (b). Sparsity of the FDFD global matrix with respect to the permittivity tensor form [18] (b). The black squares represent non-zero values to be stored in the sparse matrix. The fewer the black squares, the less demanding the memory requirement is.

One of the possibilities to represent the second-order derivatives with respect to the transverse directions is to use the three-point central finite differences (FD), allowing for the eigenvalue problem discretization (where $\Delta x$ and $\Delta y$ are the step/grid sizes in $x$ - and $y$-direction, respectively):

$$
\begin{aligned}
& \left(\frac{-2}{\Delta x^{2}}+\frac{-2}{\Delta y^{2}}+k_{0}^{2} \varepsilon\right) E_{x, y}+\left(\frac{1}{\Delta x^{2}}\right) E_{x-1, y} \\
& +\left(\frac{1}{\Delta x^{2}}\right) E_{x+1, y}+\left(\frac{1}{\Delta y^{2}}\right) E_{x, y-1} \\
& \quad+\left(\frac{1}{\square}\right) E_{x, y+1}=\beta^{2} E_{x, y} .
\end{aligned}
$$

At each point of the mesh grid in such definition, $E(x, y)$ is associated with itself and another four neighboring 
points, as represented by the global matrix shown in Fig. 1a [with nonzero elements represented by the pictograms introduced in Eq. (2)]. From the eigenvalues obtained it is possible to calculate the effective refractive indices of the modes (while $n_{\text {eff }}=\beta / k_{0}$, where $k_{0}$ is a wavevector) and the eigenvectors represent the modal field spatial patterns. The full size of the global matrix is $(M \times N)^{2}$, where $M$ and $N$ are the grid points used to represent the fiber geometry in the $x$ - and $y$-direction, respectively. Fortunately, with the development of sparse matrix packages, it is possible to reduce significantly the memory requirement for the global matrix has to be stored (see Table I).

Complexity of the FDFD eigenvalue problem and size of the global matrix (estimated in case of sparse matrices) with respect to the permitivity tensor form applied.

\begin{tabular}{l|c|c}
\hline \hline & Full & Sparse \\
\hline scalar-field & $(N \times M) \times(N \times M)$ & $N \times M \times 5$ \\
\hline isotropic (vector-field) & \multirow{3}{*}{$(N \times M \times 2) \times(N \times M \times 2)$} & $N \times M \times 2 \times 5$ \\
\cline { 1 - 1 } diagonally anisotropic & & $N \times M \times 2 \times 9$ \\
\cline { 1 - 1 } transversely anisotropic & & $N \times M \times 2 \times 16$ \\
\hline arbitrarily anisotropic & $(N \times M \times 4) \times(N \times M \times 4)$ &
\end{tabular}

It is worth mentioning that despite its simplicity, some authors still apply the scalar-field FDFD for the modeling of MOFs [23].

\subsection{Material anisotropy in FDFD}

To date different FDFD schemes allowing for a study on light propagation in optical structures with material anisotropy (characterized by different forms of permittivity tensor, as given by Eqs. (3)-(6)) have been successfully developed. Four possible forms of permittivity (second-rank) tensors, with different complexity in the numerical problem to be computed, are shown below.

$$
\begin{aligned}
& \text { isotropic } \overline{\bar{\varepsilon}}=\left[\begin{array}{lll}
\varepsilon & 0 & 0 \\
0 & \varepsilon & 0 \\
0 & 0 & \varepsilon
\end{array}\right], \\
& \text { diagonally anisotropic } \overline{\bar{\varepsilon}}=\left[\begin{array}{ccc}
\varepsilon_{x x} & 0 & 0 \\
0 & \varepsilon_{y y} & 0 \\
0 & 0 & \varepsilon_{z z}
\end{array}\right], \\
& \text { transversely anisotrpic } \overline{\bar{\varepsilon}}=\left[\begin{array}{ccc}
\varepsilon_{x x} & \varepsilon_{x y} & 0 \\
\varepsilon_{y x} & \varepsilon_{y y} & 0 \\
0 & 0 & \varepsilon_{z z}
\end{array}\right], \\
& \text { arbitrarily anisotropic } \overline{\bar{\varepsilon}}=\left[\begin{array}{ccc}
\varepsilon_{x x} & \varepsilon_{x y} & \varepsilon_{x z} \\
\varepsilon_{y x} & \varepsilon_{y y} & \varepsilon_{y z} \\
\varepsilon_{z x} & \varepsilon_{z y} & \varepsilon_{z z}
\end{array}\right] .
\end{aligned}
$$

For example, computation methods which account for the diagonal anisotropy in optical fibers [14], the transverse anisotropy in dielectric waveguides [18], and the general anisotropy in LC-core waveguides [22] have been reported. Some of the formulations are based on the curl Maxwell equations with the first-order derivatives applied in the finite difference scheme [14, 22]. It is on the contrary to other solutions in which the divergence Maxwell equations are also introduced resulting thus in the finite difference schemes with second-order deriva- tives applied [18]. Numerical scheme presented here is one based on the vector-field FDFD proposed in [14] with its simplicity and the use of the Yee staggered mesh (which reduces the number of grid points by a factor of two for a fixed fiber geometry, which is beneficial in the large and complex structures characterization) as the main motivations for this particular method application. In particular, by introducing some correction terms, we have extended the primary FDFD scheme to be capable for the transverse material anisotropy (e.g., in uniaxial nematic LC shown in Fig. 2, with elements of the permittivity tensor in uniaxial nematic LC as a function of orientation angles given in Eqs. (7) below) to be accounted.

$$
\begin{aligned}
& \varepsilon_{x x}=n_{\mathrm{o}}^{2}+\left(n_{\mathrm{e}}^{2}-n_{\mathrm{o}}^{2}\right) \sin ^{2} \phi \cos \theta, \\
& \varepsilon_{x y}=\varepsilon_{y x}=\left(n_{\mathrm{e}}^{2}-n_{\mathrm{o}}^{2}\right) \sin ^{2} \phi \sin \theta \cos \theta, \\
& \varepsilon_{x z}=\varepsilon_{z x}=\left(n_{\mathrm{e}}^{2}-n_{\mathrm{o}}^{2}\right) \sin \phi \cos \phi \cos \theta, \\
& \varepsilon_{y y}=n_{\mathrm{o}}^{2}+\left(n_{\mathrm{e}}^{2}-n_{\mathrm{o}}^{2}\right) \sin ^{2} \phi \sin ^{2} \theta, \\
& \varepsilon_{y z}=\varepsilon_{z y}=\left(n_{\mathrm{e}}^{2}-n_{\mathrm{o}}^{2}\right) \sin \phi \cos \phi \sin \theta, \\
& \varepsilon_{z z}=n_{\mathrm{o}}^{2}+\left(n_{\mathrm{e}}^{2}-n_{\mathrm{o}}^{2}\right) \cos ^{2} \phi .
\end{aligned}
$$

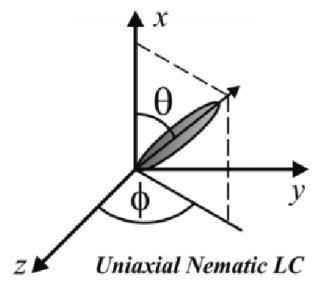

Fig. 2. Elements of the permittivity tensor in uniaxial nematic LC can be represented as a function of orientation angles $\theta$ and $\phi$.

\subsection{Vector-field FDFD}

While extending the FDFD formulation proposed by Zhu and Brown (with the staggered Yee mesh configuration applied for the finite differences incorporation) [14] 
by assuming more general form of the permittivity tensor - i.e., with the transverse material anisotropy considered, some additional cross-terms (boxed in Eqs. (9)(13)) appear in the eigenvalue equations to be solved. In

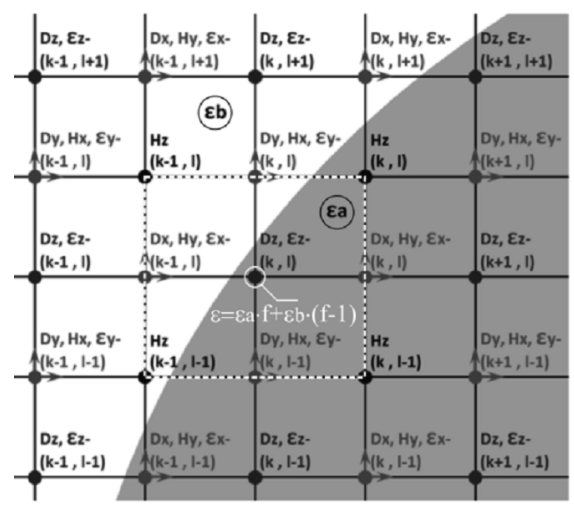

Fig. 3. The staggered Yee mesh configuration for the vector-field FDFD implemented in this work. As an example the boundary of the regions with permittivities $\varepsilon_{a}$ and $\varepsilon_{b}$ is presented, where the region with $\varepsilon_{a}$ is shaded in gray. The area enclosed by the dashed white line is considered for index-averaging procedure described in Sect. 2.5 (where $f$ is the ratio of the region with the electric permittivity $\varepsilon_{a}$ to this area).

this case, two eigenvalue equations (written in the matrix forms) with respect to the electric and magnetic field read as follows (please note that details on the algebra in arriving at the global equations were skipped due to limitations of this communication):

$$
P\left[\begin{array}{l}
E_{x} \\
E_{y}
\end{array}\right]=\left[\begin{array}{ll}
P_{x x} & P_{x y} \\
P_{y x} & P_{y y}
\end{array}\right]\left[\begin{array}{l}
E_{x} \\
E_{y}
\end{array}\right]=\beta^{2}\left[\begin{array}{c}
E_{x} \\
E_{y}
\end{array}\right],
$$

where $P$ is the global matrix for the electric field, with the sub-matrices

$$
\begin{aligned}
P_{i i} & =-\frac{1}{k_{0}^{2}} U_{i}\left(\frac{I}{\varepsilon_{z z}}\right) V_{j} V_{i} U_{j}+\left[k_{0}^{2} I+U_{i}\left(\frac{I}{\varepsilon_{z z}}\right) V_{i}\right] \\
& \left.\times\left[\varepsilon_{i i}+\frac{1}{k_{0}^{2}} V_{j} U_{j}\right]+U_{i}\left(\frac{I}{\varepsilon_{z z}}\right) V_{i} \varepsilon_{j i}\right], \\
P_{i j} & =U_{i}\left(\frac{I}{\varepsilon_{z z}}\right) V_{j}\left[\varepsilon_{i i}+\frac{1}{k_{0}^{2}} V_{i} U_{i}\right] \\
& -\frac{1}{k_{0}^{2}}\left[k_{0}^{2} I+U_{i}\left(\frac{I}{\varepsilon_{z z}}\right) V_{i}\right] V_{j} U_{i} \\
& +k_{0}^{2} \varepsilon_{i j}+U_{i}\left(\frac{I}{\varepsilon_{z z}}\right) V_{i} \varepsilon_{i j}
\end{aligned}
$$

and similarly

$$
Q\left[\begin{array}{l}
H_{x} \\
H_{y}
\end{array}\right]=\left[\begin{array}{ll}
Q_{x x} & Q_{x y} \\
Q_{y x} & Q_{y y}
\end{array}\right]\left[\begin{array}{l}
H_{x} \\
H_{y}
\end{array}\right]=\beta^{2}\left[\begin{array}{l}
H_{x} \\
H_{y}
\end{array}\right],
$$

where $Q$ is the global matrix for the magnetic field, with the sub-matrices:

$$
\begin{aligned}
Q_{i i} & =-\frac{1}{k_{0}^{2}} V_{i} U_{j} U_{i}\left(\frac{I}{\varepsilon_{z z}}\right) V_{j}+\left[\varepsilon_{j j}+\frac{1}{k_{0}^{2}} V_{i} U_{i}\right] \\
& \left.\times\left[k_{0}^{2} I+U_{j}\left(\frac{I}{\varepsilon_{z z}}\right) V_{j}\right]+\varepsilon_{j i} U_{i}\left(\frac{I}{\varepsilon_{z z}}\right) V_{j}\right], \\
Q_{i j} & =-\left[\varepsilon_{j j}+\frac{1}{k_{0}^{2}} V_{i} U_{i}\right] U_{j}\left(\frac{I}{\varepsilon_{z z}}\right) V_{i}+\frac{1}{k_{0}^{2}} V_{i} U_{j} \\
& \times\left[k_{0}^{2} I+U_{i}\left(\frac{I}{\varepsilon_{z z}}\right) V_{i}\right] \\
& +\left[-k_{0}^{2} \varepsilon_{j i}-\varepsilon_{j i} U_{i}\left(\frac{I}{\varepsilon_{z z}}\right) V\right],
\end{aligned}
$$

where $i \equiv(x, y) ; j \equiv(x, y)$ and $i \neq j$. Please note that when $\varepsilon_{x y}=\varepsilon_{y x}=0$, the sub-matrices in Eqs. (9), (10), (12), (13) reduce to those in [14]. The finite-difference sub-matrices introduced in Eqs. (9)-(13), whose form depends on how the two-dimensional fields are arranged into one-dimensional vectors for matrix computation (with the arrangement procedure identical to the field-to-vector assignment applied for the scalar-field FDFD with its global matrix shown in Fig. 1a). The form of the finite-difference sub-matrices $(U, V)$ is shown in Eqs. (14)-(17).

$$
U_{x}=\frac{1}{\Delta x}\left[\begin{array}{cccccc}
-1 & 1 & & & \\
& -1 & 1 & & & \\
& & \cdot & . & & \\
& & & \cdot & \cdot & \\
& & & -1 & 1 \\
& & & & & -1
\end{array}\right],
$$

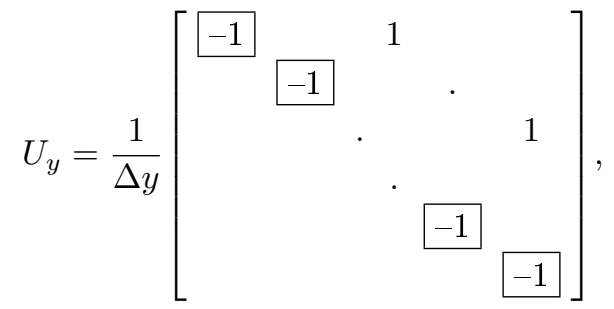

$$
V_{x}=\frac{1}{\Delta x}\left[\begin{array}{cccccc}
1 & & & & \\
-1 & 1 & & & \\
& -1 & \cdot & & \\
& & \cdot & \cdot & & \\
& & & \cdot & 1 & \\
& & & -1 & 1
\end{array}\right] \text {, }
$$

$$
V_{y}=\frac{1}{\Delta y}\left[\begin{array}{llllll}
1 & & & & \\
& 1 & & & \\
& & \cdot & & \\
& & & & \\
& & & & 1 & \\
& & & & 1
\end{array}\right] \text {. }
$$


The FD matrices also depend on the boundary conditions specified. In particular their elements boxed in Eqs. (14)-(17) may take value of zero when specific (Dirichlet and/or Neumann) boundary conditions are considered. Please note that also here there may exist zeros in the off-diagonal values of the global matrix which represent the boundaries in $x$-direction, as it was given by the gray shades in Fig. 1a.

After solving the eigenvalue problem defined as above, it is possible to obtain the spatial distributions for transverse components of the electric and magnetic field. The corresponding longitudinal components can be then calculated from the following equations:

$$
\begin{aligned}
& \mathrm{i} k_{0} H_{z}=U_{x} E_{y}-U_{y} E_{x}, \\
& -\mathrm{i} k_{0} \varepsilon_{z z} E_{z}=V_{x} H_{y}-V_{y} H_{x} .
\end{aligned}
$$

As mentioned before, the first-hand results obtained in FDFD simulations are the effective refractive index and the modal field distribution, which correspond to the eigenvalue and the eigenfield (eigenvector), respectively. It is important to note that with the vector-field scheme, it is also possible to obtain polarization information regarding the eigenfield with different parameters such as birefringence, polarization mode dispersion (PMD), polarization extinction ratio, and dichroic ratio possible to be derived.

\subsection{Improvement of FDFD performance}

In order to improve the performance of FDFD schemes, some additional (numerical) techniques can be applied. In analyzed case, the staggered Yee mesh has been already implemented in the FDFD scheme in order to reduce the number of grid points needed while maintaining high accuracy. Index-averaging is another simple but useful technique for improvement of the FDFD performance (especially for cases when the resolution for a spatial discretization is low or insufficient). It has been proven to increase greatly the convergence of the numerical simulations. The main idea of the index-averaging is to represent a spatial refractive index distribution with the use of more points, especially in the regions of the refractive index changes. In this work, index-averaging is implemented through an interpolation technique - specifically, the refractive index in a specific grid-point (of the Yee mesh applied) is approximated by the interpolation of the sub-grid points, the assignment of their values ac- cording to the original fiber geometry, and the calculation of their average. The accuracy of such approximation depends on the interpolation factor $\Gamma$, which is a positive (even) integer that specifies how many additional sub-grid points are used. In particular, when $\Gamma=N$ is applied, $(N-1)$ additional points are assigned in between the original grid points, and the refractive index profile of the area with $(N+1)$-by- $(N+1)$ points centered around the original grid point is averaged and assigned to the grid point. It means that for $\Gamma=10$ (typically applied in this paper) refractive index in each grid point is obtained as an average over 121 sub-grid points.

In addition, some spurious (non-physical) modes can be effectively eliminated from prospective solutions by performing: (i) correlation between the obtained eigenfields and the presumed modal fields; (ii) edge-cutting, which is adequate to the replacement of the silica outside the photonic lattice by air, and can be still regarded physical for the fundamental core-guiding modes.

\section{Verification and benchmarking of proposed schemes}

One method that can be applied in order to verify the correctness of the implementation of the proposed FDFD scheme is to compare the numerical results with good references, which are often analytical results. The idea is to use simple step-index fiber geometries (particularly those with low index-contrast) for which modal analyses can be performed analytically, and then extend the use of the validated numerical schemes to more complicated structures (if not solved analytically then with modal characteristics well described in literature). In addition, numerical verification of proposed schemes can be performed. The most important results of this verification method are the convergence curves which are typically represented as the functions of either the step size (grid-mesh size) or the size of the calculation window. Fast and steady convergence is expected for a good simulation scheme, as it represents smaller uncertainty of the simulation. On the other hand, although the uncertainty may be minimized, there is still no information regarding the calculation error. Similarly, good convergence against the size of the calculation window may suggest that the simulation results are intrinsic, but does not give any information on whether the intrinsic results are correct.

Parameters of the optical structures taken for the benchmarking.

TABLE II

\begin{tabular}{c|c|c|c|c}
\hline \hline Parameters & SMF & HC-SIF & Parameters & HoF \\
\hline wavelength, $\lambda$ & $1.55 \mu \mathrm{m}$ & $1.5 \mu \mathrm{m}$ & wavelength, $\lambda$ & $1.5 \mu \mathrm{m}$ \\
core diameter, $d$ & $8.2 \mu \mathrm{m}$ & $6 \mu \mathrm{m}$ & pitch, $\Lambda$ & $2.3 \mu \mathrm{m}$ \\
corerefractive index, $n_{\text {core }}$ & 1.449504 & 1.45 & hole-diameter, $d$ & $1.0 \mu \mathrm{m}$ \\
cladding refractive index, $n_{\text {clad }}$ & 1.444304 & 1 & cladding refractive index, $n_{\text {silica }}$ & 1.45 \\
refractive index reference, $\Delta n$ & $0.360035 \%$ & $45 \%$ & hole refractive index, $n_{\text {air }}$ & 1 \\
normalized frequency, $V$ & 2.038674 & 8.796459 & calculation window & $6 \Lambda=13.8 \mu \mathrm{m}$
\end{tabular}


In order to raise the confidence level of the numerical simulations performed, the proposed FDFD schemes have been tested for various fiber geometries according to the (analytical and numerical) verification methods described above. In particular, the performance of the numerical tools has been checked for: (i) a single-mode fiber (SMF), (ii) a step-index fiber with high index-contrast (HC-SIF), and (iii) photonic crystal fiber (holey fiber, HoF). Parameters of the optical structures taken for the benchmarking are specified in Table II. Results obtained are described in Sects. 3.1-3.3 and summarized in Sect. 3.5. Please note that in all cases grid-sizes in both transverse directions are assumed to be equal (i.e., $\Delta x=\Delta y$ ). An average factor $\Gamma$ of 10 is taken in all cases where refractive-index-averaging technique was applied.

\subsection{Single-mode fiber}

A standard single-mode telecommunication fiber (SMF), with parameters defined in Table II, has been considered as the first optical structure for the benchmarking. Its diameter is smaller, and its minimum radius of curvature in the structure larger, than those for a typical PCF, making the simulation conditions to be less stringent. With the refractive index-contrast which is low, the SMF can be analyzed analytically with the use of the LP approximation [12]. Specifically, effective refractive index for $\mathrm{LP}_{01}$ fiber-mode has been found to be $n_{\text {eff }}=1.446535$ (calculated with a step size of $10^{-8}$
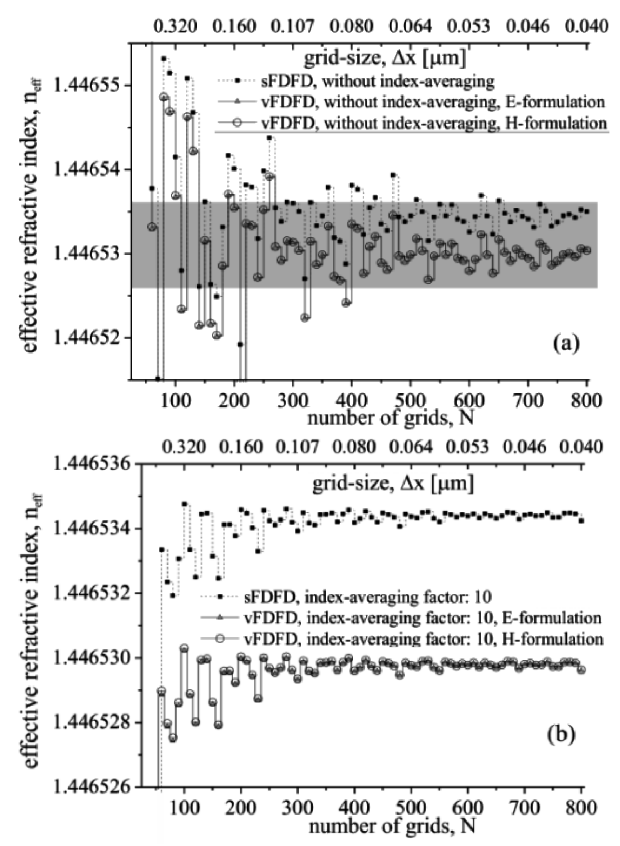

Fig. 4. Effective refractive index of the fundamental mode obtained without (a) and with (b) index averaging as a function of number of grids for fixed size of the calculation window. Results for scalar and vector (both for $E$ - and $H$-formulation) version of FDFD scheme are compared. Gray rectangle in (a) represents a range of effective refractive index from part (b). for the normalized propagation constant) and it has been taken as the reference value for the numerical simulations performed with FDFD. Convergence analyzes with respect to the grid step-size for fixed size of the calculation window $(32 \mu \mathrm{m} \times 32 \mu \mathrm{m})$ have been performed (Fig. 4a). When index-averaging technique is applied, the convergence reaches the order of $10^{-6}$ for number of grids more than 250 , while this value without index-averaging is almost $2 \times 10^{-5}$. It proves that the index-averaging technique improves largely the convergence, especially when the resolution in space discretization is low. For 250 grids, the spatial resolution is about $0.13 \mu \mathrm{m}$, which in analyzed case is roughly $1 / 12$ the wavelength. This criterion, together with convergence of at least $10^{-6}$, has been considered as a minimum requirement to be fulfilled in performed simulations. However, it should be noted that in some cases such criterions are not quite accessible due to the limited computation power (memory limitations).

By comparing the results with the analytic value for $\mathrm{LP}_{01}\left(n_{\text {eff }}=1.446535\right)$, we obtain the errors of simulations, which is $10^{-6}$ [relative error (RE) of $0.7 \mathrm{ppm}$ ] for the scalar-field FDFD, and $5 \times 10^{-6}(\mathrm{RE}=3.5 \mathrm{ppm})$ for the vector-field FDFD. Less agreement obtained for the latter ensure from the specificity of the LP approximation applied to get the reference value. It is worth to note that the $E$ - and $H$-formulations do not make any difference in the vector-field FDFD - the two curves representing them in Figs. 4a,b almost overlap with each other.

\subsection{High index-contrast step-index fiber}

While for optical fibers with a high index-contrast, the scalar approximation is no longer valid, an HC-SIF is a good candidate to show the discrepancy between the scalar- and the vector-field FDFD formulations. The fiber geometry taken into consideration (with parameters specified in Table II) is the same as one discussed in [14], where the reference value of the effective refractive index of the fundamental mode is taken as 1.438604. Setting

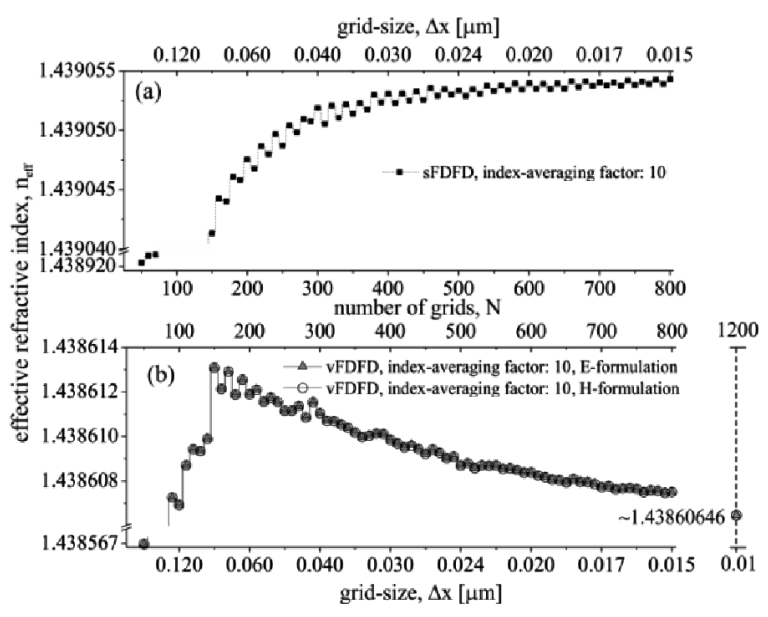

Fig. 5. Convergence curves of the effective refractive index for the fundamental mode in the HC-SIF obtained with scalar- (a) and vector-field FDFD (b). Reference value is taken as 1.438604 [14]. 
the calculation window to be $12 \mu \mathrm{m} \times 12 \mu \mathrm{m}$ (the same as in [14] to ensure that obtained results are comparable), numerical simulations were performed with both scalarand vector-field FDFDs (see Fig. 5a, b). The resultant effective refractive indices $n_{\text {eff }}$ for the fundamental mode are 1.439054 and 1.438606 (for 1200 grids), respectively.

\subsection{Holey fiber}

A photonic crystal fiber has been considered as a final optical structure for the benchmarking. A holey PCF (also described in [14]) belongs to the same type of geometry as the host PCF of the PLCF of interest. Specifically, analyzed structure is shown in Fig. 6a, with parameters specified in Table II.

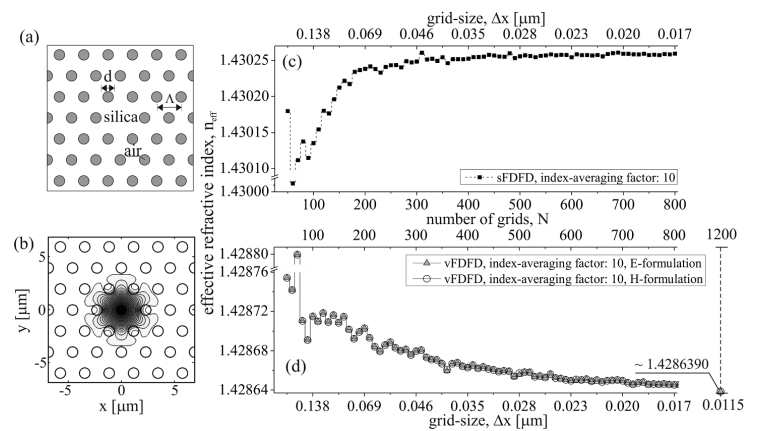

Fig. 6. Scheme of the photonic structure in the form of the holey fiber (a). Field pattern $\left(\left|E_{x}\right|\right)$ for the fundamental (core-guiding) mode propagating in the structure as an effect of the modified total internal reflection (b). Convergence curves for the fundamental core mode in the HoF obtained with scalar- and vector-field $\operatorname{FDFD}(\mathrm{c}),(\mathrm{d})$.

The convergence curves obtained at $\lambda=1.5 \mu \mathrm{m}$ for the fundamental core-guiding mode (whose spatial field distribution (i.e. absolute value for $E_{x}$ component) is presented in Fig. 6b) are shown in Fig. 6c, d. Obtained re-

sults are eventually compared with the reference values in Table III. One concern of the triangular-lattice PCFs (as analyzed here) is the degeneracy of the fundamental (core) modes. It has been shown both theoretically [24] and numerically [25] that, although there exists structural difference along the $x$ - and the $y$-directions, the fundamental modes in two orthogonal polarization states are degenerated (i.e., are characterized by the same effective refractive indices). Simulations performed with use of the FDFD schemes proposed here also confirm such degeneracy.

\subsection{Summary on benchmarking of FDFD}

To sum up, the scalar- and the vector-field FDFD schemes introduced in this work have been carefully tested with some exemplary (well-known) fiber geometries with validation concepts based on analytical and numerical verifications. The performance of the FDFD schemes implemented, with numerical calculations executed for 800 grids in each transverse direction (unless it is marked differently) and with the index-averaging technique applied is summarized in Table IV. It seems that it can be pushed even further but computational power (memory requirement, in particular) appears to be a concern regarding the convergence of the simulations.

TABLE III

Reference values of the effective refractive index for fundamental mode in comparison to simulations results obtained in this work.

\begin{tabular}{c|c|c}
\hline \hline Reference & Approach & Results \\
\hline$[26]$ & LFN & 1.42805 \\
{$[27]$} & FD-BPM & 1.42868 \\
{$[17]$} & FDFD & 1.42858 \\
{$[14]$} & FDFD & 1.42868 \\
current work & SFDF & 1.43026 \\
current work & vFDFD & 1.42864
\end{tabular}

TABLE IV

The benchmarking results for the scalar- and vector-field FDFD schemes (typically 800 grids were applied).

\begin{tabular}{|c|c|c|c|c|c|}
\hline Fiber & $\begin{array}{c}\text { Reference } \\
\text { (fundamental } \\
\text { core mode) }\end{array}$ & $\begin{array}{l}\text { Scalar-field } \\
\text { FDFD }\end{array}$ & $\begin{array}{l}\text { Relative } \\
\text { error }\end{array}$ & $\begin{array}{l}\text { Vector-field } \\
\text { FDFD }\end{array}$ & $\begin{array}{l}\text { Relative } \\
\text { error }\end{array}$ \\
\hline single-mode fiber & 1.446535 & 1.446534 & $<1 \mathrm{ppm}$ & 1.446530 & $4 \mathrm{ppm}$ \\
\hline $\begin{array}{l}\text { high index-contrast } \\
\text { step-index fiber }\end{array}$ & 1.438604 & 1.439054 & $313 \mathrm{ppm}$ & $\begin{array}{c}1.438608 \\
1.4386065^{a}\end{array}$ & $\begin{array}{c}3 \mathrm{ppm} \\
2 \mathrm{ppm}^{a}\end{array}$ \\
\hline holey fiber & 1.42805 & 1.43026 & $0.2 \%$ & $\begin{array}{c}1.428640 \\
1.428639^{a}\end{array}$ & $\begin{array}{l}413 \mathrm{ppm}^{a} \\
412 \mathrm{ppm}^{a}\end{array}$ \\
\hline
\end{tabular}

${ }^{a}$ for 1200 grids.

In the tests performed, the limitations of the scalar-field formulation have been shown quantitatively, as well as its difference to the vector-field calculation, and the errors of the FDFDs compared to analytical values. The proposed FDFD schemes give satisfactory results for the single-mode fiber, HC-SIF, and photonics crystal fiber 
geometries which were tested. Specifically, when vector-field FDFD is applied, relative error of $2 \mathrm{ppm}$ is observed for $n_{\text {eff }}$ of the fundamental mode in HC-SIF. For the holey fiber geometry, the result of the vector-field FDFD differs by $6 \times 10^{-4}(\mathrm{RE}=413 \mathrm{ppm})$ from that of the localized function method [26]. Moreover, it should be noted that for complex structures results obtained with scalar-field FDFD have larger errors, with 313 ppm for HC-SIF and $0.2 \%$ for $\mathrm{HoF}$, which are approximately 100 times and 5 times larger than with vector-field FDFD, respectively.

\section{Results for the PLCF of interest}

\subsection{Structure definition and preliminary experimental tests}

The PCF chosen for our studies is LC $13 / 7$, with three rings of the air-holes, manufactured at the Maria Curie-Skłodowska University (UMCS) in Lublin, Poland. The optical microscope image of the structure is shown in Fig. 7a. We have decided to use a 3-ring PCF because of its relatively small transverse extent and thus the possibility to include the entire periodic structure within calculation window. 6CHBT (also known as 6CPS) has been selected to be the LC for infiltration. The chemical structure of $6 \mathrm{CHBT}$ is presented as an inset in Fig. $7 \mathrm{~b}$ and its main optical properties can be found in [28]. While the values of refractive indices (both $n_{\mathrm{o}}$ and $n_{\mathrm{e}}$ ) of LC used for infiltration are higher than that of the silica $\left(n_{\text {silica }}\right)$, guiding core modes (i.e., localized within the silica core) of the analyzed structure are obtained due to the photonic bandgap-guiding mechanism.

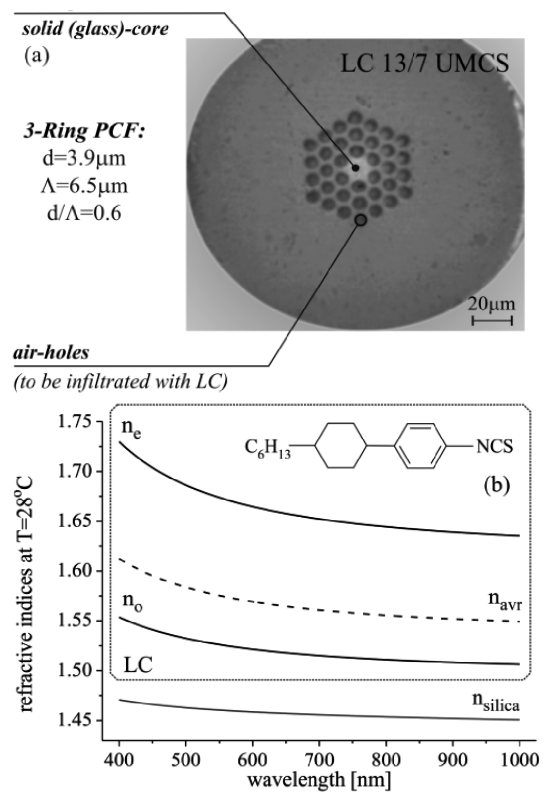

Fig. 7. The optical microscope image of the three-ring host PCF (a). The refractive indices of the $6 \mathrm{CHBT}$ nematic LC [28] at $28^{\circ} \mathrm{C}(\mathrm{b})$, where $n_{\mathrm{avr}}=2 n_{\mathrm{o}} / 3+$ $n_{\mathrm{e}} / 3$ is taken when random orientation of LCs within PCF structure is considered. Refractive index of silica $\left(n_{\text {silica }}\right)$ at the same temperature [29] is also shown for comparison.

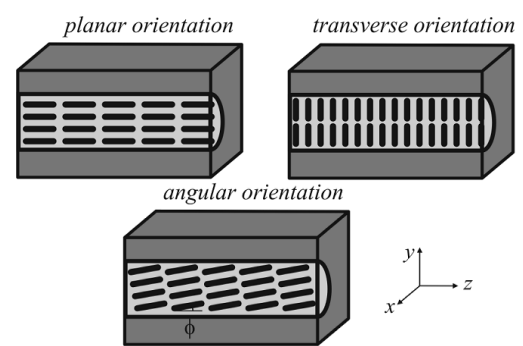

Fig. 8. Different molecular arrangements of LC inside the air-holes of PCF. Corresponding values of permittivity tensor elements are given in Eqs. (20)-(22).

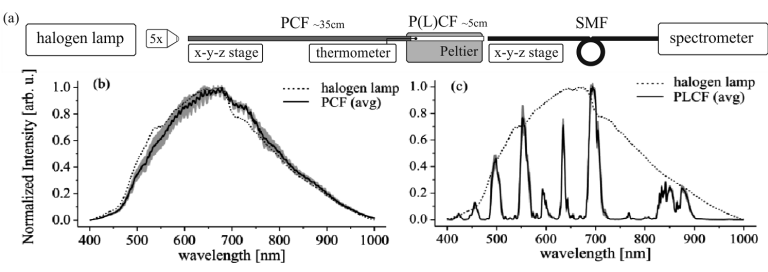

Fig. 9. Experimental setup for measuring the transmission spectra of $\mathrm{P}(\mathrm{L}) \mathrm{CF}$ (a). Obtained results representing normalized (and averaged) transmission spectrum for empty PCF (b) and for PLCF (c). Dashed lines show the normalized spectrum of the light source (together with the probing single-mode fiber of spectrometer). The gray shade in graphs (b)-(c) corresponds to the uncertainty of the measurements.

After introducing the liquid crystal into the air-holes of the host PCF, several possible molecular arrangements can be obtained (e.g., when proper orientation treatment is performed [30] or/and under external fields [4-8]) as shown in Fig. 8. Otherwise, if molecular orientation is not predictable - it is often assumed to be random and the average refractive index $n_{\text {avr }}$ of LC may be considered (for calculations).

The experimental setup for measuring the spectral characteristics of $\mathrm{P}(\mathrm{L}) \mathrm{CFs}$ is shown in Fig. 9a. A white light from a continuous spectrum (in the range of 300$1050 \mathrm{~nm}$ ) source (Ocean Optics Tungsten Halogen Calibration Light Source HL-2000-CAL and HL-2000-CAL-ISP) was focused onto the PCF input-facet by means of a microscope objective. A probing SMF was then attached in proximity to the fiber end-facet and connected to the spectrometer. The signal was analyzed by a fiber optics spectrometer (Ocean Optics HR4000) with spectral resolution of around $0.25 \mathrm{~nm}$. A Peltier module was used to heat up or cool down the PLCF sample. A Testo 735 precise thermometer with resolution of $0.05^{\circ} \mathrm{C}$ and accuracy of $0.1^{\circ} \mathrm{C}$ was used to monitor temperature changes.

Transmission spectra measured for PCF before and after infiltration are shown in Figs. 9b,c, respectively. As one can see, the transmission spectrum of an empty PCF is continuous, while several transmission peaks within the spectral range of the light source exist for the PLCF sample. Such selectivity on wavelengths to be transmitted 
through the photonic structure is characteristic for the PBG-guiding mechanism of propagation.

Above results and observations can be confirmed numerically when suitable simulations are performed over specific range of wavelengths. Effective refractive index of the fundamental core mode with respect to wavelength for PCF and PLCF are shown in Figs. 10b and 11, respectively. However, it has to be noted that owing to the limit of experimental means, direct quantitative comparison is not quite accessible. Instead, qualitative comparison of the experimental and theoretical results may be performed.

\subsection{Numerical results}

Following the idea of the numerical verification introduced in Sect. 3, the auto-validation of the numerical schemes (i.e. the convergence analysis) for the host (empty) PCF structure has been performed (for $\lambda=$ $686 \mathrm{~nm}$ ) as shown in Fig. 10a. In particular, both scalarand vector-field FDFD have been introduced, with a calculation window of $50 \mu \mathrm{m} \times 50 \mu \mathrm{m}$ used in simulations. Using the reference value for the fundamental effective refractive index (obtained by fitting the convergence curve with respect to the square of the grid-size), the simulations convergence to about $1 \mathrm{ppm}$ has been achieved for vector-field FDFD with 800 grids. Eventually, vector-field FDFD (with a grid-size of $0.625 \mu \mathrm{m}$ ) was applied for the specific spectral range (similar to that used in experimental conditions), with an edge-cutting performed in order to reduce a number of spurious results.

In the analyzed case effective refractive index of the fundamental mode vs. wavelength is represented by continuous function (see Fig. 10b). It is worth to note that the first two modes [with the highest (and identical) effective refractive indices] are degenerated upon polarization. Such degeneracy has been reported both theoretically [24] and numerically [25] in literature. It has been confirmed that results of the analogous simulations performed with use of commercial software (COMSOL Multiphysics), based on the finite elements methods are quantitatively and qualitatively consistent.

Vector-field FDFD has been applied for the PLCF sample with the same simulation parameters as shown in Fig. 10b. In addition, the isotropic form of the permittivity tensor for LC (with values of $n_{\text {avr }}^{2}$ on its diagonal) has been used, following thus an assumption of no orientation order of the LC molecules within the PCF.

Figure 11 shows the numerical results on the effective refractive index of the fundamental core mode of the PLCF sample as a function of wavelength. In the simulations performed different number (from 10 to 100) of the sequential eigenvalues (with the corresponding eigenfields/eigenvectors) has been searched. The core-guiding modes of each wavelength have been then manually inspected and their effective refractive indices have been extracted.

It is worth mentioning here that PBG-guiding modes here have very different field patterns (see insets in the

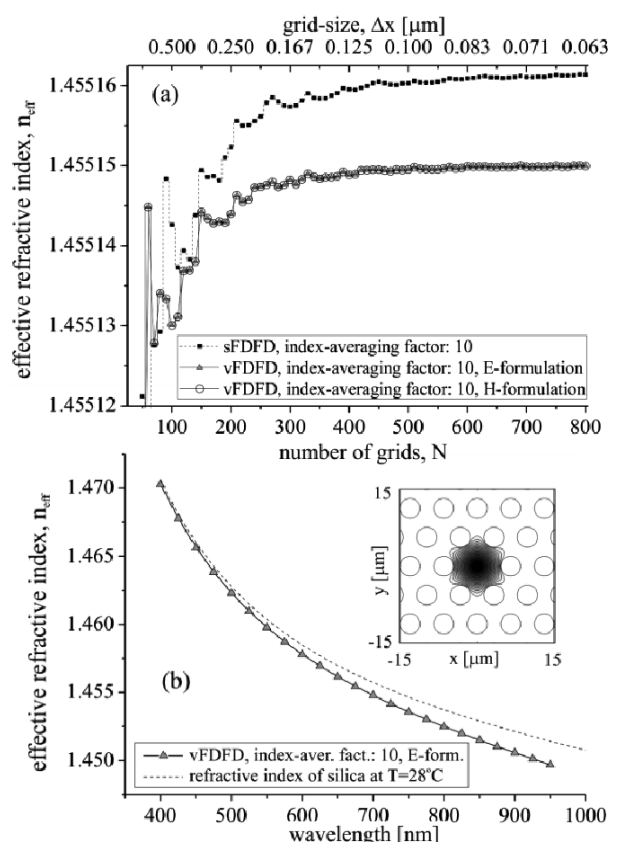

Fig. 10. Convergence curves of the effective refractive index $n_{\text {eff }}$ (at $\lambda=686 \mathrm{~nm}$ ) for the fundamental core-guiding mode (with a spatial field pattern shown in the upper right hand corner) in the $\mathrm{PCF}$ with respect to the number of grids calculated with scalar-field FDFD (black squares) and vector-field FDFD (represented by light-gray triangles and hollow circles for $E$ - and $H$-field formulation, respectively) (a). Effective refractive index of the fundamental mode in the PCF as a function of the wavelength (b). Dispersion curve for the silica at $T=$ $28^{\circ} \mathrm{C}[29]$ is represented by a dashed line for comparison.

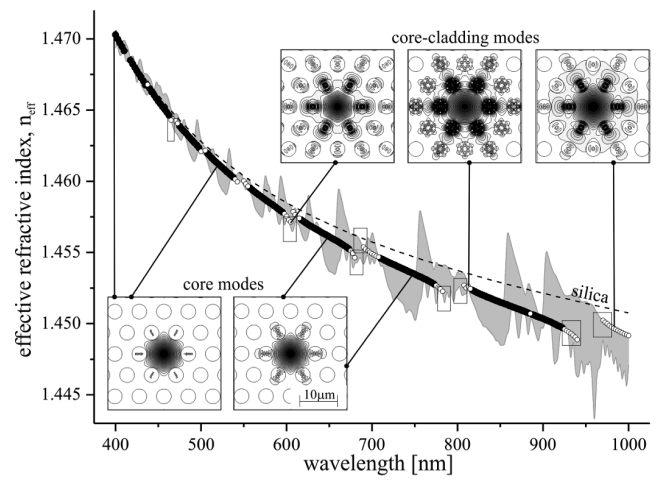

Fig. 11. Numerical results on the effective refractive index of the fundamental (quasi- $x$-polarized) core-guiding mode in the PLCF sample as a function of wavelength (black points). Hollow circles represent the cases in which modal area exceeds significantly a core region (and light is guided also in the PCF holes infiltrated with LC) and are marked with boxes if imaginary part of refractive index occurs. The eigenvalue range of simulations is shaded in gray. Insets show eigenfields (more precisely $\left|E_{x}\right|$ is plotted in the central part of the calculation window) for some selected wavelengths. 
lower left hand corner of Fig. 11) when compared to those of the empty PCF host as shown in Fig. 10b. By analyzing other eigenfields obtained in simulations and shown as insets in Fig. 11, one can see that in case of PLCF, in addition to the core-guiding modes and the typical cladding modes (when light propagates in the silica cladding) which are obtained for empty PCF, some hole-guiding modes (called here as the cladding modes as well; see Fig. 11) are also observed.

Such possibilities of different solutions to be obtained in simulations further increase the number of spurious results when searching for the core-guiding modes. Unlike the index-guiding empty $\mathrm{PCF}$, the eigenfield patterns of the PBG-guiding PLCF sample change significantly with wavelength, and for some of them, there are no core-guiding modes within the simulation range (represented by the light-gray shade in Fig. 11, shows the range of the eigenvalues obtained in simulations). In the view of the transmission spectrum, such lack of the core-guiding modes coincides with discrete transmission gaps in experiment.

Results obtained suggest that in general the two polarizations are not degenerated (which was confirmed by analogous simulations performed with use of COMSOL software). However, the difference between effective refractive indices of two quasi- $x$ - and quasi- $y$-polarized modes (in order of magnitude of from $10^{-8}$ to $10^{-7}$ for core-guiding modes calculated with use of 800 grids) is small but it is still impossible to unambiguously conclude on the degeneracy.

It is important to note that effective refractive indices for two orthogonal polarizations can be obtained when calculated with vector-field FDFD. It means that information on the birefringence and polarization mode dispersion of the specific structure can be obtained. It also allows for the identification of the hybrid-guiding, which is obtained for some configurations when index-guiding is observed for one and PBG-guiding for the other state of polarization, as reported in $[31,32]$. In analyzed case of PLCF structures, it is possible to align the liquid crystal molecules within the fiber starting from the planar and ending up with the transverse configuration (which are specific cases of the angular orientation as shown in Fig. 8), as it was suggested by some previous reports showing the significant tunability of PLCFs with external fields, e.g. [8]. While the vector-field FDFD scheme implemented in this work allows transverse anisotropy to be accounted, it is possible to study the effects of rotation of LC molecules (in the transverse plane) on the propagation properties of the PLCF considered. Permittivity tensor elements for different molecular arrangements of LC inside the air-holes of PCF (see Fig. 8) are:

for planar orientation

$$
\varepsilon_{x x}=n_{\mathrm{o}}^{2}, \varepsilon_{y y}=n_{\mathrm{o}}^{2}, \varepsilon_{z z}=n_{\mathrm{e}}^{2}, \varepsilon_{i j(i \neq j)}=0,
$$

for transverse orientation

$$
\varepsilon_{x x}=n_{0}^{2}, \varepsilon_{y y}=n_{\mathrm{e}}^{2}, \varepsilon_{z z}=n_{0}^{2}, \varepsilon_{i j(i \neq j)}=0,
$$

$$
\begin{aligned}
& \varepsilon_{x x}=n_{0}^{2}, \varepsilon_{y y}=n_{\mathrm{o}}^{2} \cos ^{2} \phi+n_{\mathrm{e}}^{2} \sin ^{2} \phi, \\
& \varepsilon_{z z}=n_{\mathrm{e}}^{2} \cos ^{2} \phi+n_{\mathrm{o}}^{2} \sin ^{2} \phi, \varepsilon_{i j(i \neq j)}=0 .
\end{aligned}
$$

Exemplary results of simulations performed for different orientational angles $(\phi, \theta)$ of the LC molecules, with permittivity tensor calculated with formulae given in Eqs. (20)-(22) and refractive indices of LC shown in Fig. 7b, are summarized in Table $\mathrm{V}$.

\section{TABLE V}

\begin{tabular}{|c|c|c|c|c|}
\hline$\lambda[\mathrm{nm}]$ & $\begin{array}{l}\text { LC conf., } \\
\theta=90^{\circ}\end{array}$ & $n_{\mathrm{eff}, x}$ & $n_{\mathrm{eff}, y}$ & Remark \\
\hline \multirow{8}{*}{$775 \mathrm{~nm}$} & $\begin{array}{c}\text { no LC } \\
\text { (empty PCF) }\end{array}$ & 1.45302447 & 1.45302447 & degenerated \\
\hline & $\phi=0^{\circ}$ & 1.45303678 & 1.45313378 & $\Delta=9.7 \times 10^{-5}$ \\
\hline & $\phi=15^{\circ}$ & 1.45303748 & 1.45313371 & $\Delta=3.4 \times 10^{-5}$ \\
\hline & $\phi=30^{\circ}$ & 1.45300314 & 1.45300314 & degenerated \\
\hline & $\phi=45^{\circ}$ & 1.45300822 & 1.45300822 & degenerated \\
\hline & $\phi=60^{\circ}$ & 1.45300286 & 1.45300286 & degenerated \\
\hline & $\phi=75^{\circ}$ & 1.45293748 & 1.45298277 & $\Delta=4.5 \times 10^{-5}$ \\
\hline & $\phi=90^{\circ}$ & 1.453133809 & 1.45304586 & $\Delta=8.8 \times 10^{-5}$ \\
\hline $800 \mathrm{~nm}$ & $\phi=90^{\circ}$ & - & 1.45284146 & $\begin{array}{c}\text { single } \\
\text { polarization }\end{array}$ \\
\hline $650 \mathrm{~nm}$ & $\phi=75^{\circ}$ & - & - & no core mode \\
\hline
\end{tabular}

Exemplary results of the vector-field FDFD simulations performed for different molecular orientation of LC within PLCF sample (with transverse anisotropy considered).

From the results obtained it may be concluded that it is possible to find wavelengths (e.g., $775 \mathrm{~nm}$ in Table V) for which continuous tuning of the molecular orientation is possible with a core-guiding mode always present in the structure. For some specific settings (i.e., wavelength and sample configuration), there are cases when core-guiding mode appear only for one polarization state or it does not propagate in the structure. It is worth to note that for $\theta=90^{\circ}$ and $\phi=30^{\circ}, 45^{\circ}, 60^{\circ}$ analyzed structure behaves like the empty one (i.e., there are no gaps in $n_{\text {eff }}(\lambda)$ curve and modes are degenerated upon polarization) with the only difference in the values of effective refractive index of the core-guiding modes (which are higher when LC is present in the PCF sample).

\section{Conclusions}

In this work, we focus on numerical methods for the characterization of PLCFs with accessible computational effort. More specifically, both the scalar- and the vector-field schemes were formulated theoretically and implemented numerically with the use of the finite difference methods (FDFD/FM). It has been found that compared to analytical values, the relative error introduced in the scalar-field formulation is almost 100 times higher than the vector-field formulation for a HC-SIF. When vector-field FDFD is applied, relative error of 3 ppm is observed for effective refractive index of the fundamental mode in HC-SIF and 413 ppm in a HoF with index-guiding geometry. The $<0.1 \%$ relative errors qualify for simulations on PLCFs. However, spurious results appear to be a concern when PBG guiding fiber geometries are considered, as the eigenvalue range of the simulation is very limited under some conditions. Simulations show the wavelength selectivity in transmission spectrum, which is correspondingly 
observed in experiments. Future examinations on both the experiment and the numerical schemes are required to justify the simulations and to analyze the discrepancy. It is worth to underline that a vector-field FDFD scheme developed and implemented here can easily take into consideration the anisotropy of LC with arbitrary rotation in the transverse plane with respect to the propagation axis of the fiber.

\section{Acknowledgments}

K. Rutkowska would like to acknowledge Homing Plus Programme granted by the Foundation for Polish Science (contract number HOMING PLUS/2010-2/11) and co-financed by the European Regional Development Fund.

\section{References}

[1] D.B. Eggleton, Ch. Kerbage, P. Westbrook, R. Windeler, A. Hale, Opt. Exp. 9, 698 (2001).

[2] P.St.J. Russell, Science 299, 358 (2003).

[3] C. Knight, Nature 424, 847 (2003).

[4] T.R. Woliński, K. Szaniawska, K. Bondarczuk, P. Lesiak, A.W. Domański, R. Dąbrowski, E. Nowinowski-Kruszelnicki, Opto-Electron. Rev. 13, 59 (2005).

[5] T.R. Woliński, K. Szaniawska, S. Ertman, P. Lesiak, A.W. Domański, R. Dabrowski, E. Nowinowski-Kruszelnicki, J. Wójcik, Meas. Sci. Technol. 17, 985 (2006).

[6] F. Du, Y.-Q. Lu, S.-T. Wu, Appl. Phys. Lett. 85, 2181 (2004).

[7] T.T. Larsen, A. Bjarklev, D.S. Hermann, J. Broeng, Opt. Exp. 11, 2589 (2003).

[8] S. Ertman, T.R. Woliński, J. Beeckman, K. Neyts, P.J.M. Vanbrabant, R. James, F.A. Fernández, Acta Phys. Pol. A 118, 1113 (2010).

[9] A. Czapla, W.J. Bock, T.R. Woliński, R. Dąbrowski, E. Nowinowski-Kruszelnicki, Acta Phys. Pol. A $\mathbf{1 2 0}$ 589 (2011).

[10] T.R. Woliński, S. Ertman, D. Budaszewski, M. Chychłowski, A. Czapla, R. Dąbrowski, A.W. Domański, P. Mergo, E. Nowinowski-Kruszelnicki, K.A. Rutkowska, M. Sierakowski, M. Tefelska, Phot. Lett. Poland 3, 20 (2011).

[11] C.-H. Lee, C.-H. Chen, C.-L. Kao, C.-P. Yu, S.-M. Yeh, W.-H. Cheng, T.-H. Lin, Opt. Exp. 18, $2814(2010)$
[12] D. Gloge, Appl. Opt. 10, 2252 (1971).

[13] K. Saitoh, M. Koshiba, J. Lightwave Technol. 23 3580 (2005)

[14] Z. Zhu, T.G. Brown, Opt. Exp. 10, 853 (2002).

[15] K. Bierwirth, N. Schulz, F. Arndt, IEEE Trans. Microwave Theory Techn. MTT-34, 1104 (1986).

[16] M.S. Stern, IEEE Proc. 135, 56 (1988).

[17] P. Lüsse, P. Stuwe, J. Schule, H.-G. Unger, J. Lightwave Technol. 12, 487 (1994).

[18] A.B. Fallahkhair, K.S. Li, T.E. Murphy, J. Lightwave Technol. 26, 1423 (2008)

[19] K.S. Yee, IEEE Trans. Antennas Propag. AP-14, $302(1966)$

[20] S. Guo, F. Wu, S. Albin, R. Rogowski, Opt. Exp. 12 , 1741 (2004).

[21] C.-P. Yu, H.-C. Chang, Opt. Exp. 12, 6165 (2004).

[22] M.-Y. Chen, S.-M. Hsu, H.-C. Chang, Opt. Exp. 17, 5965 (2009).

[23] J. Riishede, N.A. Mortensen, Jesper Lægsgaard, J. Opt. A: Pure Appl. Opt. 5, 534 (2003).

[24] M.J. Steel, T.P. White, C.M. Sterke, R.C. McPhedran, L.C. Botten, Opt. Lett. 26, 488 (2001).

[25] M. Koshiba, K. Saitoh, IEEE Phot. Techn. Lett. 13, 1313 (2001)

[26] D. Mogilevtsev, T.A. Birks, P.S.J. Russell, J. Lightwave Technol. 17, 2078 (1999).

[27] W.P. Huang, C.L. Xu, IEEE J. Quantum Electron. 29, 2639 (1993).

[28] J. Schirmer, P. Kohns, T. Schmidt-Kaler, A. Muravski, S. Yakovenko, V. Bezborodov, R. Dabrowski, P. Adomenas, Mol. Cryst. Liq. Cryst. 307, 17 (1997).

[29] M. Medhat, S.Y. El-Zaiat, A. Radi, M.F. Omar, J. Opt. A: Pure Appl. Opt. 4, 174 (2002).

[30] M.S. Chychłowski, S. Ertman, M.M. Tefelska, T.R. Woliński, E. Nowinowski-Kruszelnicki, O. Yaroshchuk, Acta Phys. Pol. A 118, 1100 (2010).

[31] J. Sun, C.C. Chan, J. Opt. Soc. Am. B 24, 2640 (2007).

[32] D.C. Zografopoulos, E.E. Kriezis, J. Lightwave Technol. 27, 773 (2009). 